\title{
Atomic and Molecular Phases of the Interstellar Medium
}

\author{
Mordecai-Mark Mac Low
}

Dept. of Astrophysics, American Museum of Natural History, Central Park West at 79th Street, New York, NY 10024-5192, USA and Institut für Theoretische Astrophysik, Zentrum für Astronomie der Universität Heidelberg, Albert-Ueberle-Str. 2, 69121 Heidelberg, Germany email: mordecai@amnh.org

\begin{abstract}
This review covers four current questions in the behavior of the atomic and molecular interstellar medium. These include whether the atomic gas originates primarily in cold streams or hot flows onto galaxies; what the filling factor of cold gas actually is in galactic regions observationally determined to be completely molecular; whether molecular hydrogen determines or merely traces star formation; and whether gravity or turbulence drives the dynamical motions observed in interstellar clouds, with implications on their star formation properties.
\end{abstract}

Keywords. astrochemistry, hydrodynamics, MHD, turbulence, shock waves, ISM: general, ISM: structure, ISM: molecules, ISM: kinematics and dynamics

\section{Introduction}

Star formation occurs primarily in atomic and molecular interstellar gas, although Keto (2002) emphasizes that around a massive enough star, even ionized gas can be accreted. In this review, I discuss a number of recent advances in our understanding of the origin and dynamics of that gas and its relation to star formation.

\section{Cold Accretion and its Limits}

The origin of this gas lies in gas accreted onto galaxies during their formation and subsequent evolution. Perspectives on how that gas arrives have changed several times recently. Originally, it was thought that all gas accreted into the halo of a galaxy the size of the Milky Way would be heated to the virial temperature of over $10^{6} \mathrm{~K}$, followed by gradual cooling and accretion. However, a decade ago, numerical simulations started to suggest that a major, possibly even dominant alternative was for dense streams to accrete from the cosmic web, dense enough to cool quickly when compressed and thus to never heat to the virial temperature (e.g. Fardal et al. 2001; Birnboim \& Dekel 2003; Kereš et al. 2005; 2009; Ocvirk et al. 2008; Dekel \& Birnboim 2006; Dekel et al. 2009; 2013; Goerdt et al. 2015). However, Nelson et al. (2013), using the AREPO Voronoi mesh code (Springel 2010), argued that cold streams are likely only important for small galaxies at high redshift (see Fig. 1), while their appearance at lower redshifts and for larger galaxies is a numerical artifact of the smoothed particle hydrodynamics (SPH) method, as exemplified in that paper by GADGET (Springel 2005).

Evidence for continuing accretion of gas in the modern Milky Way can be gained from observations of high velocity clouds of neutral gas falling towards the galactic plane at velocities over $70 \mathrm{~km} \mathrm{~s}^{-1}$. Material falling in at slower velocities has been shown to have Galactic chemical abundances, while the high velocity clouds have far lower abundances, suggesting extragalactic origin, as reviewed by Putman et al. (2012). Simple analytic 


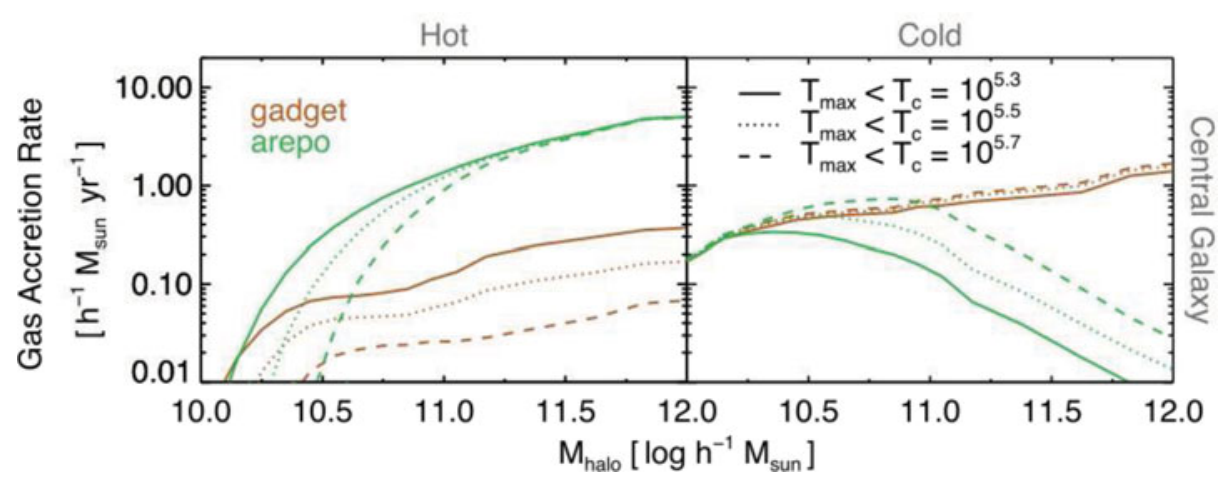

Figure 1. Comparison of gas accretion rates for hot gas (left, with temperature above $T_{c}$ as shown in legend) and cold gas (right) as a function of halo mass, for SPH models (brown) and Voronoi mesh models (green). The two methods agree for small masses typical of high redshifts, but the Voronoi mesh code predicts most gas will reach high temperatures for large halo sizes, disagreeing with the SPH results. Adapted from Nelson et al. (2013).

models modeled on the flow of water into a bathtub and down the drain can guide our understanding of the cycle of gas into the atomic phase from infalling ionized gas, and then out of it again through star formation or expulsion of the gas in galactic outflows (Bouché et al. 2010; Dekel et al. 2013; Dekel \& Mandelker 2014).

\section{Why a Cold, Dense, ISM is Mostly Hot}

The thermodynamic state of the atomic and diffuse molecular gas is controlled by the balance between optically-thin radiative cooling and photoelectric heating by far ultraviolet radiation. Between around $100 \mathrm{~K}$ and $10^{4} \mathrm{~K}$, the cooling curve is a sufficiently steep function of temperature to produce a region of thermal instability (Field et al. 1969, Wolfire et al. 1995, 2003). In the case of pressures typical of the Milky Way, this leads to a two-phase medium (solid line in Fig. 2). When pressures increase (dotted line), only the cold, dense phase remains thermally stable.

Ostriker et al. (2010) argued that, in fact, this transition leads to self-regulation of star formation. The pressure range within which a warm phase will be present is determined by the far ultraviolet radiation field produced by stars. If the vertical hydrostatic pressure exceeds that range, it will drive nearly all the gas into the cold phase, promoting star formation, and thus increasing the far ultraviolet field until a warm medium can be reestablished. Thus, the combination of thermal and hydrostatic equilibrium controls, in the terms of the bathtub model, how fast the bathtub drains.

While the point that high enough pressures produce a predominantly cold temperature distribution is widely understood, what is perhaps less appreciated is the resulting spatial distribution of the gas. Gatto et al. (2015) and Li et al. (2015) emphasize that the filling factor of the cold, dense gas will be small in such a high-pressure environment. In regions with supernova explosions (either from active star formation or even Type Ia supernovae from older populations) the space in between the cold, dense clumps is filled with hot, tenuous gas (see Fig. 3), the third phase of McKee \& Ostriker (1977).

Observational tracers of atomic and molecular gas will show that the mass of the interstellar gas is almost entirely in cold, molecular material, leading to the description of the region as a fully molecular interstellar medium. The hot gas, on the other hand, is difficult to observe, as its density is low and temperature high enough that it emits primarily in bremsstrahlung. However, it still completely dominates the volume filling 


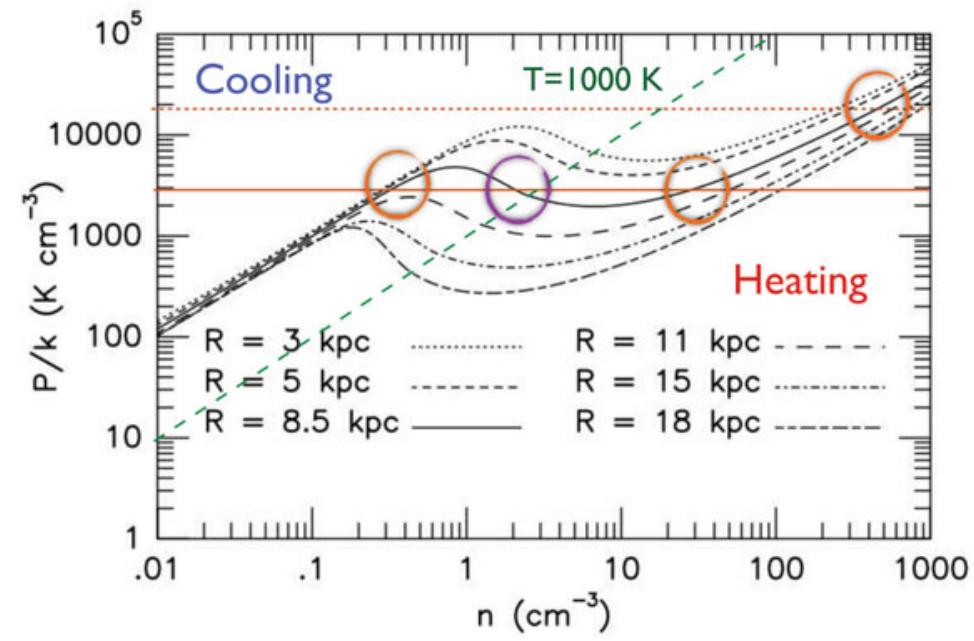

Figure 2. Thermal equilibrium curves at different radii in the Milky Way, compared to a typical pressure near the Solar circle (solid orange line), and compared to the pressure in the molecular ring or starburst galaxies (dashed orange line). Orange circled points represent stable equilibria, where small perturbations of temperature (motions perpendicular to the green-dashed line, with increasing temperature towards the left) result in restoring increases in cooling or heating, as labeled. Purple circled point is unstable. Adapted from Wolfire et al. (2003).
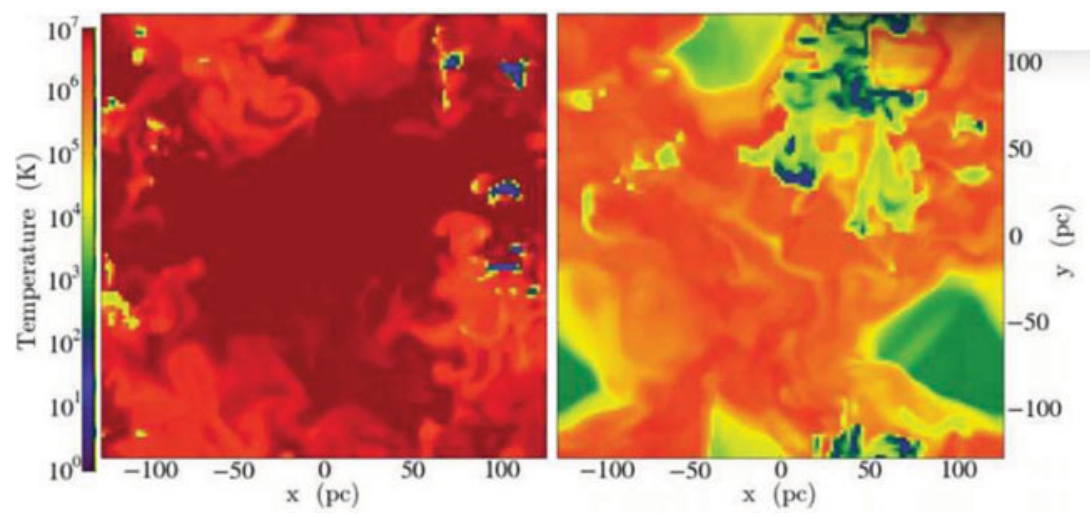

Figure 3. Temperature distributions on slices through periodic boxes of supernova-driven turbulence. On the left, supernovae explode in random positions, while on the right, half the supernovae explode in density peaks, substantially increasing the loss of energy to radiative cooling. On the left, pressures have reached high enough levels to eliminate the warm stable phase. Adapted from Gatto et al. (2015).

factor, and needs to be taken into account in any model of high-pressure, fully molecular, interstellar gas. Joung et al. (2009) demonstrated that following the Kennicutt-Schmidt law for star formation in stratified disks results in a three-phase medium, while Walch et al. (2015) showed that increasing the star formation rate by a factor of three already leads to a cold interstellar medium filled with hot gas.

\section{Molecules are a Consequence of Star Formation}

Observations have demonstrated a linear correlation between the surface density of star formation and that of molecular hydrogen (Rownd \& Young 1999; Wong \& Blitz 2002; 
Bigiel et al. 2008; Leroy et al. 2008). This raises the question of whether this correlation implies a causal relationship. Is molecular hydrogen formation required for star formation to occur?

It has more recently become evident that many tracers of high density gas correlate just as well with the star formation rate as does molecular hydrogen. For example, the tracer molecule HCN was shown by Gao \& Solomon (2004) to also correlate with star formation rate in both normal spiral galaxies and luminous and ultraluminous infrared galaxies. In the solar neighborhood, the number of young stellar objects in a cloud correlates with the mass of the cloud contained in regions with a K-band infrared extinction $A_{K}>0.8$ (Lada et al. 2010). Clark \& Glover 2014 indeed found thresholds for star formation in numerical simulations that reproduce this observed threshold. However, the physical process producing this threshold is not molecule formation, but rather dust shielding from heating by the background far ultraviolet radiation.

Indeed Krumholz et al. (2011) and Glover \& Clark (2012) have demonstrated that atomic line cooling is as effective as molecular cooling in bringing gas down to star forming temperatures of 10-20 K. Figure 4 demonstrates that the same cold, star-forming gas appears in numerical simulations with full molecular and atomic cooling; with only atomic cooling, but still including molecular hydrogen formation; and with all molecular physics suppressed, but still including dust shielding from far ultraviolet heating. Only the removal of dust shielding from the simulation suppresses star formation.

Thus, it appears that the formation of molecular hydrogen does not control star formation. Rather, it seems, star formation, or more generally gravitational collapse of atomic gas, controls molecular hydrogen formation.

\section{Molecular Cloud Dynamics Driven by Gravity Rather Than Turbulence}

Molecular cloud dynamics offer a useful window to their origins. The argument has been made that they form by the sweeping up of smaller clouds in superbubbles or spiral arms (e.g. Tasker \& Tan 2009). An alternative proposal is that clouds form from large scale gravitational collapse, as proposed by Hartmann et al. (2001) to explain the apparent correlation of star formation across entire molecular clouds.

Models of the supernova-driven, stratified interstellar medium can be used to examine these scenarios. One example of such a model derives from work by Joung \& Mac Low (2005), Joung et al. (2009), and Hill et al. (2012). It includes both clustered and random supernovae, a background gravitational potential from the stellar disk and the halo, photoelectric heating and equilibrium ionization cooling, and magnetic fields. These models swept up gas into filaments that appeared similar to large molecular clouds. An example of such a filament is shown in top panel of Figure 5 .

Ibáñez-Mejía et al. (2015) added the self-gravity of the gas to these models, turning it on after a full vertical galactic fountain had been set up over the course of over $200 \mathrm{Myr}$. This long preparation period also allowed plenty of time for the dense regions to come into equilibrium with the supernova-driven turbulence in the surrounding diffuse medium. However, when the self-gravity was turned on, immediate collapse ensued, as is shown in the example of Figure 5. One immediate and notable result was the enhancement of the filamentary morphology to the extreme levels characteristic of observed infrared dark clouds (e.g., Simon et al. 2006).

Within $5 \mathrm{Myr}$, this collapse leads to the formation of unresolved dense cores within which rapid star formation appears inevitable. As these first models have no mechanism to simulate the local prompt feedback in ionizing radiation, stellar winds and so forth 


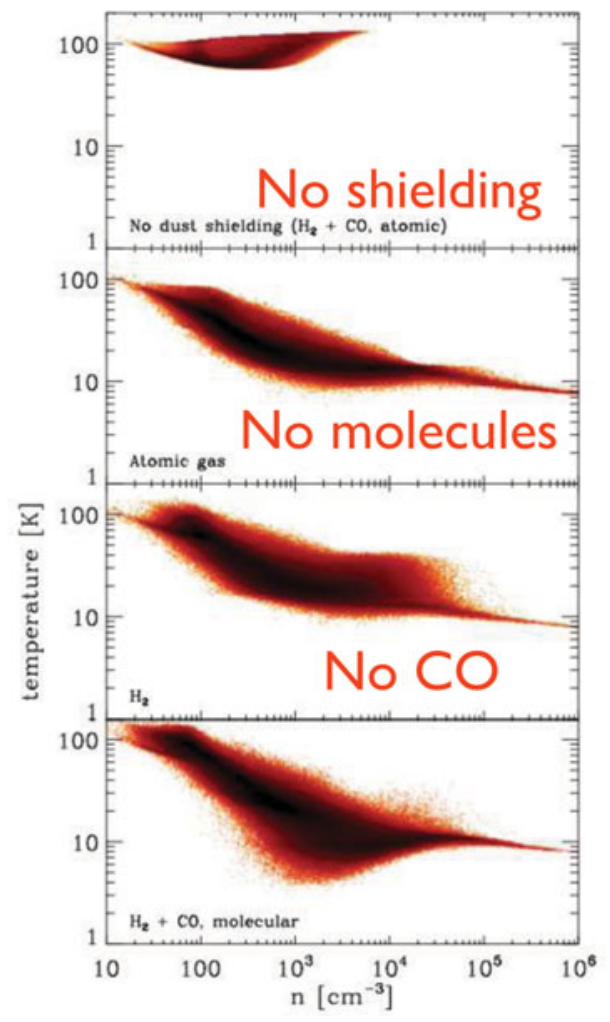

Figure 4. Distribution of temperature and pressure in models of turbulent, atomic gas including both atomic and molecular chemistry and cooling processes, as well as photoelectric heating modulated by dust shielding. The bottom panel shows the full model, while the upper panels have successively less physics. The first panel from the bottom neglects molecular line cooling, the second also neglects molecular hydrogen formation, and the top also neglects dust shielding. Gas reaches $10 \mathrm{~K}$ and high densities in all but the last of these models. Adapted from Glover \& Clark (2012).

from this star formation, we can no longer follow its evolution after this point. However it is clear already that without this local feedback, star formation would reach unrealistic rates, just as first argued by Zuckerman \& Palmer (1974).

In order to understand this rapid collapse and fragmentation, it is necessary to examine the dynamics of these dense regions. Ibáñez-Mejía et al. (2015) identified clouds using density contours and demonstrated that these clouds quite uniformly had velocity dispersions $\sigma<1 \mathrm{~km} \mathrm{~s}^{-1}$, even at radii $R>30 \mathrm{pc}$, quite different from the Larson (1981) size-velocity dispersion relation. This is shown by the yellow dots in all three panels of Figure 6 , in contrast to the observed relation presented as the dashed line, which is a fit to the subset of the Galactic Ring Survey (Jackson et al. 2006) presented in Heyer et al. (2009). These low velocity dispersions cannot resist gravitational collapse, and indeed the clouds do collapse, forming the same sort of filamentary structures familiar from the free gravitational collapse of the cosmic web. Although magnetic fields are present in the simulation, they are unable to effectively resist this collapse. As they collapse, their observed velocity dispersions increase to the observed values.

Ballesteros-Paredes et al. (2011) emphasize that the relationship between surface density, velocity dispersion, and radius expected from freely falling material differs by only 


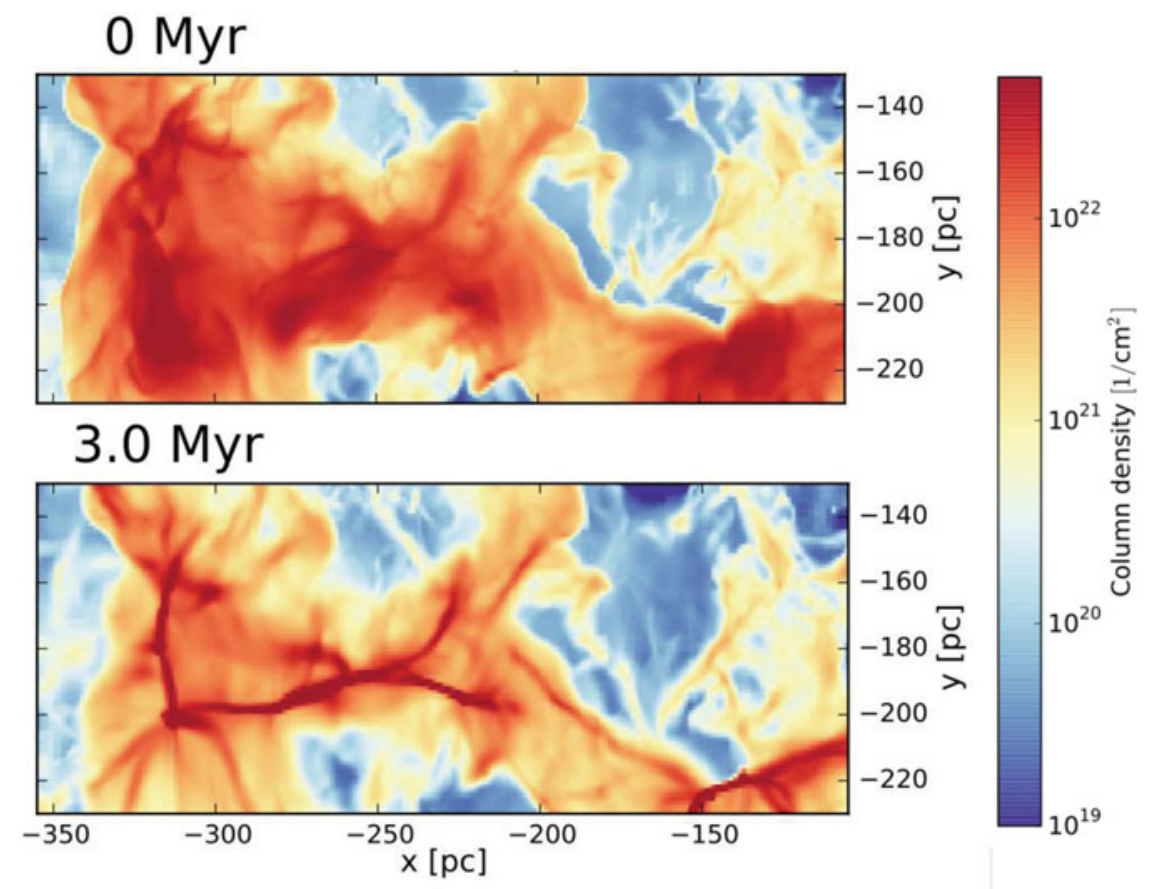

Figure 5. Vertical projection through a sub-region of a supernova-driven, stratified, magnetized model of the interstellar medium at times of 0 and 3 Myr after self-gravity has been turned on. (The full domain is a $1 \times 1 \times 20 \mathrm{kpc}$ vertical cut through the galactic disk.) Prompt collapse occurs, forming filaments strongly resembling observed IRDCs, with star-forming cores strung along them. Adapted from Ibáñez-Mejía et al. (2015).

a factor of $\sqrt{2}$ from that of material in virial equilibrium, and both appear consistent with the observations as summarized by Heyer et al. (2009).

It is worth noting that in a periodic box simulation of supernova-driven turbulence, neglecting stratification, but run at higher resolution, Padoan et al. (2015) find velocity dispersions after 6 Myr that agree with our results. However Padoan (priv. comm., 2015) finds similar velocity dispersions in their model even prior to the onset of self-gravity, in contradiction to our results. Further investigation will be needed to establish if the difference is due to their higher resolution or their neglect of stratification and confinement within the periodic box.

\section{Summary}

In this review, I have addressed a few current topics in the theory of the atomic and molecular interstellar medium. I have focused on the following questions, with my current best guesses as to the answers.

- Do cold streams contribute to gas accretion onto galaxies only at early times and for small galaxies, or far more generally as was originally claimed? The former appears to be the result of the numerical simulations most likely to be reliable.

- How small is the actual filling factor of dense, molecular gas in an interstellar medium when it is observationally determined to be completely molecular? Quite small, with the rest of the volume occupied by low-density, hot gas with temperatures exceeding $10^{6} \mathrm{~K}$.

- Does molecular hydrogen drive star formation, or rather does star formation produce molecular hydrogen and other high density tracers as a by-product? Molecular hydrogen 


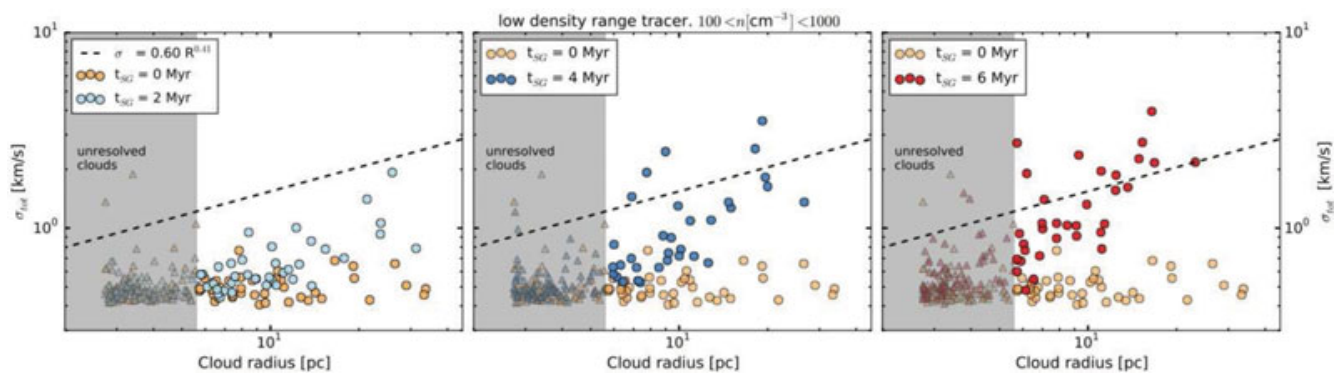

Figure 6. Radius-velocity dispersion relation for clouds drawn from the models prior to the action of self-gravity (yellow dots in all three panels), and for clouds at times of 2 Myr (left), $4 \mathrm{Myr}$ (center) and $6 \mathrm{Myr}$ (right) after self-gravity begins to act. For comparison, the observed relation derived from the data presented by Heyer et al. (2009) is shown by the dashed line. The shaded region shows clouds under 10 zones whose velocity dispersion is likely to be strongly suppressed by numerical viscosity. Adapted from Ibáñez-Mejía et al. (2015).

and other molecules appear to function far more as a tracer than a cause of star formation, particularly since atomic line cooling is nearly as effective as molecular line cooling.

- Are the observed velocity dispersions in molecular clouds caused by supernova-driven turbulence, or by gravitational collapse? Gravitational collapse seems more likely, but this question does not yet appear to be settled.

\section{References}

Ballesteros-Paredes, J., Hartmann, L. W., Vázquez-Semadeni, E., Heitsch, F., \& Zamora-Avilés, M. A. 2011, MNRAS, 411, 65

Bigiel, F., Leroy, A., Walter, F., et al. 2008, AJ, 136, 2846

Birnboim, Y. \& Dekel, A. 2003, MNRAS, 345, 349

Bouché, N., Dekel, A., Genzel, R., et al. 2010, ApJ, 718, 1001

Clark, P. C. \& Glover, S. C. O. 2014, MNRAS, 444, 2396

Dekel, A. \& Birnboim, Y. 2006, MNRAS, 368, 2

Dekel, A., Birnboim, Y., Engel, G., et al. 2009, Nature, 457, 451

Dekel, A. \& Mandelker, N. 2014, MNRAS, 444, 2071

Dekel, A., Zolotov, A., Tweed, D., et al. 2013, MNRAS, 435, 999

Fardal, M. A., Katz, N., Gardner, J. P., et al. 2001, ApJ, 562, 605

Field, G. B., Goldsmith, D. W., \& Habing, H. J. 1969, ApJ (Letters), 155, L149

Gao, Y. \& Solomon, P. M. 2004, ApJ, 606, 271

Gatto, A., Walch, S., Mac Low, M.-M. et al. 2015, MNRAS, 449, 1057

Glover, S. C. O. \& Clark, P. C. 2012, MNRAS, 421, 9

Goerdt, T., Ceverino, D., Dekel, A., \& Teyssier, R. 2015, MNRAS, 454, 637

Hartmann, L., Ballesteros-Paredes, J., \& Bergin, E. A. 2001, ApJ, 562, 852

Heyer, M., Krawczyk, C., Duval, J., \& Jackson, J. M. 2009, ApJ, 699, 1092

Hill, A. S., Joung, M. R., Mac Low, M.-M., et al. 2012, ApJ, 761, 189

Ibáñez-Mejía, J. C., Mac Low, M.-M., Klessen, R. S., \& Baczynski, C. 2015, ApJ, submitted, arXiv: 1511.05602

Jackson, J. M., Rathborne, J. M., Shah, R. Y., et al. 2006, ApJ (Suppl.), 163, 145

Joung, M. K. R. \& Mac Low, M.-M. 2006, ApJ, 653, 1266

Joung, M. R., Mac Low, M.-M., \& Bryan, G. L. 2009, ApJ, 704, 137

Kereš, D., Katz, N., Weinberg, D. H., \& Davé, R. 2005, MNRAS, 363, 2

Kereš, D., Katz, N., Fardal, M., Davé, R., \& Weinberg, D. H. 2009, MNRAS, 395, 160

Keto, E. 2002, ApJ, 580, 980

Krumholz, M. R., Leroy, A. K., \& McKee, C. F. 2011, ApJ, 731, 25

Lada, C. J., Lombardi, M., \& Alves, J. F. 2010, ApJ, 724, 687 
Leroy, A. K., Walter, F., Brinks, E., et al. 2008, AJ, 136, 2782

Li, M., Ostriker, J. P., Cen, R., Bryan, G. L., \& Naab, T. 2015, ApJ, 814, 4

Nelson, D., Vogelsberger, M., Genel, S., et al. 2013, MNRAS, 429, 3353

Ocvirk, P., Pichon, C., \& Teyssier, R. 2008, MNRAS, 390, 1326

Ostriker, E. C., McKee, C. F., \& Leroy, A. K. 2010, ApJ, 721, 975

Padoan, P., Pan, L., Haugbølle, T., \& Nordlund, A. 2015, ApJ, submitted, arXiv:1509.04663

Putman, M. E., Peek, J. E. G., \& Joung, M. R. 2012, ARAA, 50, 491

Rownd, B. K. \& Young, J. S. 1999, AJ, 118, 670

Simon, R., Rathborne, J. M., Shah, R. Y., Jackson, J. M., \& Chambers, E. T. 2006, ApJ, 653, 1325

Springel, V. 2005, MNRAS, 364, 1105

Springel, V. 2010, MNRAS, 401, 791

Tasker, E. J. \& Tan, J. C. 2009, ApJ, 700, 358

Walch, S., Girichidis, P., Naab, T., et al. 2015, MNRAS, 454, 238

Wolfire, M. G., McKee, C. F., Hollenbach, D., \& Tielens, A. G. G. M. 1995, ApJ, 453, 673

Wolfire, M. G., McKee, C. F., Hollenbach, D., \& Tielens, A. G. G. M. 2003, ApJ, 587, 278

Wong, T. \& Blitz, L. 2002, ApJ, 569, 157 